\title{
On the spectrum of high-energy cosmic rays produced by supernova remnants in the presence of strong cosmic-ray streaming instability and wave dissipation
}

\author{
V. S. Ptuskin ${ }^{1}$ and V. N. Zirakashvili ${ }^{1,2}$ \\ ${ }^{1}$ Institute for Terrestrial Magnetism, Ionosphere and Radiowave Propagation, 142190 Troitsk, Moscow Region, Russia \\ e-mail: vptuskin@izmiran.rssi.ru \\ 2 Max-Planck-Institut für Kernphysik, 69029 Heidelberg, Postfach 103980, Germany \\ e-mail: zirak@mpimail.mpi-hd.mpg.de
}

Received 23 June 2004 / Accepted 16 August 2004

\begin{abstract}
The cosmic-ray streaming instability creates strong magnetohydrodynamic turbulence in the precursor of a SN shock. The level of turbulence determines the maximum energy of cosmic-ray particles accelerated by the diffusive shock acceleration mechanism. In this paper we present the continuation of previous work (Ptuskin \& Zirakashvili 2003). We assume that Kolmogorov type nonlinear wave interactions together with ion-neutral collisions restrict the amplitude of the random magnetic field. As a result, the maximum energy of the accelerated particles strongly depends on the age of a SNR. The average spectrum of cosmic rays injected in the interstellar medium in the course of the adiabatic SNR evolution (the Sedov stage) is approximately $Q(p) p^{2} \propto p^{-2}$ at energies larger than $10-30 \mathrm{GeV} /$ nucleon and with a maximum particle energy that is close to the position of the knee in the cosmic-ray spectrum observed at $\sim 4 \times 10^{15} \mathrm{eV}$. At an earlier stage of SNR evolution - the ejecta-dominated stage described by the Chevalier-Nadyozhin solution, the particles are accelerated to higher energies and have a rather steep power-law distribution. These results suggest that the knee may mark the transition from the ejecta-dominated to the adiabatic evolution of SNR shocks which accelerate cosmic rays.
\end{abstract}

Key words. ISM: supernova remnants - ISM: cosmic rays

\section{Introduction}

Diffusive shock acceleration is considered as the main mechanism of acceleration of galactic cosmic rays. The diffusion coefficient $D(E)$, which is a function of energy, determines the maximum energy that particles can gain in the process of acceleration by the shock moving through the turbulent interstellar medium. The condition of efficient acceleration is $D(E) \leq$ $\varkappa u_{\mathrm{sh}} R_{\mathrm{sh}}$, where $R_{\mathrm{sh}}$ is the radius and $u_{\mathrm{sh}}$ is the velocity of a spherical shock, and the constant $\varkappa \sim 0.1$ (see Drury et al. 2001, and Malkov \& Drury 2001 for a review). The Bohm value of the diffusion coefficient $D_{B}=v r_{\mathrm{g}} / 3$ ( $v$ is the particle velocity, and $r_{\mathrm{g}}$ is the particle Larmor radius), which is a lower boundary of the diffusion along the average magnetic field, gives the maximum particle energy $E_{\max } \sim 2 \times 10^{14} Z\left(\mathcal{E}_{51} / n_{0}\right) \mathrm{eV}$ at the time of transition from the ejecta-dominated stage to the stage of adiabatic evolution of SNRs (the particle charge is $Z e$ ). Here we consider a SN burst with a kinetic energy of the ejecta $\mathcal{E}=\mathcal{E}_{51} \times 10^{51}$ erg in a gas with density $n_{0} \mathrm{~cm}^{-3}$ and an interstellar magnetic field $B_{0}=5 \mu \mathrm{G}$. This value of $E_{\max }$ is close but somewhat less than the energy of the "knee", the break in the total cosmic ray spectrum observed at $\sim 4 \times 10^{15} \mathrm{eV}$.
Analyzing the early stage of SNR evolution when the shock velocity is high, $u_{\mathrm{sh}} \sim 10^{4} \mathrm{~km} \mathrm{~s}^{-1}$, Bell \& Lucek (2001) found that the cosmic-ray streaming instability in the shock precursor can be so strong that the amplified field $\delta B \geq 100 \mu \mathrm{G}$ far exceeds the interstellar value $B_{0}$. The maximum particle energy increases accordingly. The cosmic-ray streaming instability is less efficient as the shock velocity decreases with time, and the nonlinear wave interactions reduce the level of turbulence at the late Sedov stage (Völk et al. 1988; Fedorenko 1990). This leads to fast diffusion and to a corresponding decrease of $E_{\max }$. The effect is aggravated by possible wave damping produced by the ion-neutral collisions (Bell 1978; Drury et al. 1996). The acceleration of cosmic rays and their streaming instability in a wide range of shock velocities was considered in our previous paper (Ptuskin \& Zirakashvili 2003, Paper I). The analytical expressions for the cosmic ray diffusion coefficient and for the instability growth rate were generalized to the case of an arbitrary strong random magnetic field, $\delta B \gtrless B_{0}$. The rate of nonlinear wave interactions was assumed to correspond to the Kolmogorov nonlinearity of magnetohydrodynamic waves. The collisional dissipation was also taken into account. The maximum energy of the accelerated particles was determined as a function of shock velocity and thus as a 
function of SNR age. The maximum energy of a particle with charge $Z e$ can be as high as $10^{17} \mathrm{Z} \mathrm{eV}$ in some very young SNRs and falls to about $10^{10} \mathrm{Z} \mathrm{eV}$ at the end of the adiabatic (Sedov) stage. The widely accepted estimate of the cosmic ray diffusion coefficient at the strong shock that corresponds to the Bohm diffusion value calculated for the interstellar magnetic field strength turns out to be incorrect. This result may explain why SNRs with an age of more than a few thousand years are not prominent sources of very high energy gammarays (Buckley et al. 1998; Aharonian et al. 2002). The presence of a strongly amplified random magnetic field in young SNRs is evidently supported by the interpretation of data on synchrotron X-ray emission from young SNRs, see e.g. Vink (2003) for a review.

The main objective of the present work is the calculation of the average spectrum of cosmic rays ejected into the interstellar medium by a SNR in a course of its evolution. Some necessary results of Paper I are presented in Sect. 2, the evolution of SNR shocks is discussed in Sect. 3, the average cosmic-ray source spectrum is calculated in Sect. 4 followed by the discussion in Sect. 5, the conclusion is given in Sect. 6. Appendix A describes the thin shell approximation used in our calculations.

\section{Maximum energy of accelerated particles}

In the test particle approximation, the momentum distribution of accelerated particles for high Mach number shocks has the canonical form $f(p) \sim p^{-4}$ (Krymsky 1977; Bell 1978). In the case of efficient acceleration, the action of cosmic ray pressure on the shock structure causes a nonlinear modification of the shock that changes the shape of the particle spectrum, making it flatter at ultrarelativistic energies (Eichler 1984; Berezhko et al. 1996; Malkov \& Drury 2001). Because of this effect, we assume that the distribution of ultrarelativistic particles at the shock is of the form $f_{0}(p) \sim p^{-4+a}$ where $0<a<0.5$; the value $a=0.3$ is used in the numerical estimates below. The normalization of $f(p)$ is such that the integral $N=4 \pi \int \mathrm{d} p p^{2} f(p)$ gives the number density of cosmic rays. The differential cosmic ray intensity is $I(E)=f(p) p^{2}$. We assume that the cosmic ray pressure at the shock is some fraction $\xi_{\text {cr }}<1$ of the upstream momentum flux entering the shock front, so that $P_{\mathrm{cr}}=\xi_{\mathrm{cr}} \rho u_{\mathrm{sh}}^{2}$, and the equation for the distribution function of relativistic accelerated particles at the shock is

$f_{0}(p, t)=\frac{3 \xi_{\mathrm{cr}} \rho u_{\mathrm{sh}}^{2} H\left(p_{\max }(t)-p\right)}{4 \pi c(m c)^{a} \varphi\left(p_{\max }\right) p^{4-a}}$,

where $p_{\max }$ is the maximum momentum of the accelerated particles, $H(p)$ is the step function, and $\varphi\left(p_{\max }\right)=\int_{0}^{p_{\max } / m c} \frac{\mathrm{d} y y^{a}}{\sqrt{1+y^{2}}}$. The approximation of the last integral at $p_{\max } \gg m c$ is $\varphi(p) \approx$ $a^{-1}(p / m c)^{a}-a^{-1}(1+a)^{-1}$. The value of $\xi_{\text {cr }} \approx 0.5$ and the total compression ratio at the shock close to 7 were found in the numerical simulations of strongly modified SN shocks by Berezhko et al. (1996). Here and below we mainly consider protons as the most abundant cosmic ray component. For ions with charge $Z$, the equations should be written as functions of $p / Z$ instead of $p$. In particular, the maximum momentum of nuclei with charge $Z$ is a factor of $Z$ larger than that of protons. We use the notation $m$ for the proton mass. The acceleration in old SNRs $\left(t \gtrsim 3 \times 10^{4}-10^{5} \mathrm{yr}\right)$ when $p_{\max } / m c<10$ is not considered in the present paper because Eq. (1) is not applied at low Mach numbers, see Paper I for details. (Using the test particle approximation for a non-modified shock, Drury et al. (2003) found that the spectrum of the accelerated particles is somewhat steeper if the diffusion coefficient increases with time compared to the case of constant $D$. This effect is not included in our consideration.)

The following steady-state equation determines the energy density $W(k)$ ( $k$ is the wave number) of the magnetohydrodynamic turbulence amplified by the streaming instability in the cosmic-ray precursor upstream of the supernova shock:

$u \nabla W(k)=2\left(\Gamma_{\mathrm{cr}}-\Gamma_{1}-\Gamma_{\mathrm{nl}}\right) W(k)$.

Here the 1.h.s. describes the advection of turbulence by a highly supersonic gas flow. The terms on the r.h.s. of the equation describe respectively the wave amplification by cosmic ray streaming, the linear damping of waves in the background plasma, and the nonlinear wave-wave interactions that may limit the amplitude of the turbulence. The equation for the wave growth rate at the shock

$$
\Gamma_{\mathrm{cr}}(k)=\frac{C_{\mathrm{cr}}(a) \xi_{\mathrm{cr}} u_{\mathrm{sh}}^{3} k^{1-a}}{\left(1+A_{\mathrm{tot}}^{2}\right)^{(1-a) / 2} c V_{\mathrm{a}} \varphi\left(p_{\mathrm{max}}\right) r_{\mathrm{g} 0}^{a}}
$$

was suggested in Paper I as the generalization of the equation derived for the case of a weak random field (Berezinskii et al. 1990). Here $V_{\mathrm{a}}=B_{0} / \sqrt{4 \pi \rho}$ is the Alfvén velocity ( $\rho$ is the gas density), $A=\delta B / B_{0}$ is the dimensionless wave amplitude, and $r_{\mathrm{g} 0}=m c^{2} / e B_{0}$. The ion-neutral and electron-ion collisions usually determine the linear damping processes in the thermal space plasma. The Kolmogorov-type nonlinearity with a simplified expression

$\Gamma_{\mathrm{nl}}=\left(2 C_{\mathrm{K}}\right)^{-3 / 2} V_{\mathrm{a}} k A(>k) \approx 0.05 V_{\mathrm{a}} k A(>k)$

at $C_{\mathrm{K}}=3.6$ (as follows from the numerical simulations by Verma et al. 1996) was used in Paper I. The wave-particle interaction is of resonant character and the resonance condition is $k_{\text {res }} r_{\mathrm{g}}=\sqrt{1+A_{\mathrm{tot}}^{2}}$, where the Larmor radius $r_{\mathrm{g}}=$ $p c / Z e B_{0}$ is defined through the regular field $B_{0}$, and $A_{\text {tot }}$ is the total amplitude of the random field. The particle scattering leads to a cosmic-ray diffusion with diffusion coefficient $D=\left(1+A_{\text {tot }}^{2}\right)^{1 / 2} v r_{\mathrm{g}}\left[3 A^{2}\left(>k_{\mathrm{res}}\right)\right]^{-1}$.

Equation (2) allows finding the following approximate equation for the dimensionless amplitude of the total random magnetic field produced by the cosmic-ray streaming instability at the shock (Paper I):

$$
\begin{aligned}
& \frac{3 u_{\mathrm{sh}}^{2} A_{\mathrm{tot}}^{2}}{2 v\left(1+A_{\mathrm{tot}}^{2}\right)}+\frac{V_{\mathrm{a}} A_{\mathrm{tot}}}{\left(2 C_{\mathrm{K}}\right)^{3 / 2}}= \\
& \frac{C_{\mathrm{cr}}(a) \xi_{\mathrm{cr}} u_{\mathrm{sh}}^{3}}{c V_{\mathrm{a}} \varphi\left(p_{\max }\right)\left(p_{\max } / m c\right)^{-a} \sqrt{1+A_{\mathrm{tot}}^{2}}} .
\end{aligned}
$$


To study the effect of nonlinear interactions, the term with linear damping in Eq. (2) was omitted in Eq. (5); $C_{\mathrm{cr}}(a)=27[4(5-a)(2-a)]^{-1}$. The maximum particle momentum satisfies the equation

$\frac{p_{\mathrm{max}}}{m c}=\frac{3 \varkappa A_{\mathrm{tot}}^{2} u_{\mathrm{sh}} R_{\mathrm{sh}}}{\sqrt{1+A_{\mathrm{tot}}^{2}} v r_{\mathrm{g} 0}}$

In the high velocity limit, when $u_{\mathrm{sh}} \gg 4 a C_{\mathrm{cr}} \xi_{\mathrm{cr}} c\left[9\left(2 C_{\mathrm{K}}\right)^{3 / 2}\right]^{-1}$ and $u_{\mathrm{sh}} \gg 3 V_{\mathrm{a}}\left[2 a C_{\mathrm{cr}} \xi_{\mathrm{cr}}\right]^{-1}$, the advection term dominates over the nonlinear dissipation term in the 1.h.s. of Eq. (5) and the wave amplitude is large, $A_{\text {tot }} \gg 1$. The maximum momentum of accelerated particles and the amplified magnetic field are then given by the approximate equations

$\frac{p_{\max }}{m c} \approx 2 \varkappa a C_{\mathrm{cr}} \xi_{\mathrm{cr}} u_{\mathrm{sh}}^{2} R_{\mathrm{sh}}\left(r_{\mathrm{g} 0} V_{\mathrm{a}} c\right)^{-1}$,

and

$A_{\mathrm{tot}} \approx \frac{2 u_{\mathrm{sh}}}{3 V_{a}} a C_{\mathrm{cr}} \xi_{\mathrm{cr}}$

Here the cosmic ray diffusion coefficient depends on the particle Larmor radius as $D \propto v r_{\mathrm{g}}^{1-a}$ at $p \leq p_{\max }$.

In the low velocity limit, when $u_{\mathrm{sh}} \ll\left[4 V_{\mathrm{a}}^{3} c^{2}\left(\pi a C_{\mathrm{cr}} \times\right.\right.$ $\left.\left.\left(2 C_{\mathrm{K}}\right)^{3} \xi_{\mathrm{cr}}\right)^{-1}\right]^{1 / 5}$ and $u_{\mathrm{sh}} \ll\left[V_{\mathrm{a}}^{2} c\left(a C_{\mathrm{cr}}\left(2 C_{\mathrm{K}}\right)^{3 / 2} \xi_{\mathrm{cr}}\right)^{-1}\right]^{1 / 3}$, the nonlinear dissipation term dominates over the advection term in the 1.h.s. of Eq. (5) and the wave amplitude is small, $A_{\text {tot }} \ll 1$. The maximum momentum of accelerated particles and the amplified magnetic field are then given by the approximate equations

$\frac{p_{\max }}{m c} \approx 24 \varkappa a^{2} C_{\mathrm{cr}}^{2} C_{\mathrm{K}}^{3} \xi_{\mathrm{cr}}^{2} u_{\mathrm{sh}}^{7} R_{\mathrm{sh}}\left(r_{\mathrm{g} 0} V_{\mathrm{a}}^{4} c^{3}\right)^{-1}$,

and

$A_{\mathrm{tot}} \approx a C_{\mathrm{cr}}\left(2 C_{\mathrm{K}}\right)^{3 / 2} \xi_{\mathrm{cr}} u_{\mathrm{sh}}^{3}\left(c V_{\mathrm{a}}^{2}\right)^{-1}$

assuming that this value of the amplified field exceeds the value of the random interstellar magnetic field. The cosmic ray diffusion coefficient depends on the particle Larmor radius as $D \propto v r_{\mathrm{g}}^{1-2 a}$ at $p \leq p_{\max }$. (Note the misprint in the numerical coefficient in the first equality of Eq. (19) in Paper I that is analogous to the present Eq. (9).)

Figure 1 illustrates the results of calculations of $p_{\max }$ at the Sedov stage of SNR evolution at $\mathcal{E}=10^{51} \mathrm{erg}$ in the warm interstellar gas with temperature $T=8 \times 10^{3} \mathrm{~K}$, average density $n_{0}=0.4 \mathrm{~cm}^{-3}$, which includes small interstellar clouds, intercloud density $n=0.1 \mathrm{~cm}^{-3}$, number density of ions $n_{\mathrm{i}}=0.03 \mathrm{~cm}^{-3}$, interstellar magnetic field strength $B_{0}=5 \mu \mathrm{G}$, see Paper I. The time dependence of the shock radius and the shock velocity are given by the following equations (the Sedov solution; see e.g. Ostriker \& McKee 1988):

$R_{\mathrm{sh}}=4.3\left(\mathcal{E}_{51} / n_{0}\right)^{1 / 5} t_{\mathrm{Kyr}}^{2 / 5} \mathrm{pc}$,

$u_{\mathrm{sh}}=1.7 \times 10^{3}\left(\mathcal{E}_{51} / n_{0}\right)^{1 / 5} t_{\mathrm{Kyr}}^{-3 / 5} \mathrm{~km} \mathrm{~s}^{-1}$,

where we assume that the ultrarelativistic gas of cosmic rays mainly determines the pressure behind the shock. The value

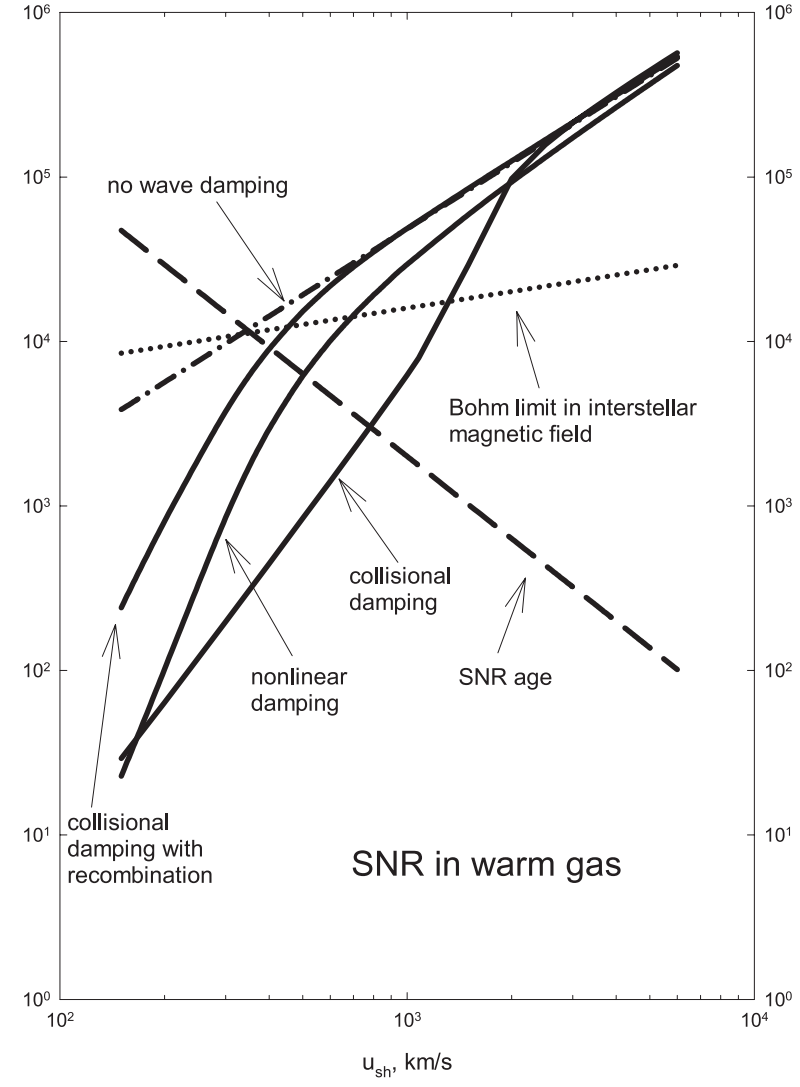

Fig. 1. The maximum momentum of accelerated protons $p_{\max }$ in units $m c$ as a function of shock velocity $u_{\mathrm{sh}}$ at the Sedov stage of supernova remnant evolution in warm interstellar gas. The three solid lines correspond to three cases of wave dissipation considered separately: nonlinear wave interactions; damping by ion-neutral collisions at constant density of neutral atoms; damping by ion-neutral collisions when the diffuse neutral gas restores its density after complete ionization by the radiation from the supernova explosion. The dashed line represents the age of a supernova remnant $t$ (plotted on the right ordinate) as a function of shock velocity. The dotted line shows the Bohm limit on maximum particle momentum calculated for the interstellar magnetic field strength. The dash-dot line gives the maximum particle momentum when the wave dissipation is not taken into account.

$x=0.04$ was assumed in the calculations in Fig. 1 . The three solid lines correspond to the three cases of wave dissipation considered separately: nonlinear wave interactions; damping by ion-neutral collisions at constant gas density; damping by ion-neutral collisions when the diffuse neutral gas restores its density after complete ionization by the radiation from the SN burst. For the last two curves, the dissipation of waves due to the ion-neutral collisions with damping rate

$\Gamma_{1}=\frac{v_{\text {in }}}{2}\left(1+\left(1+A_{\text {tot }}^{2}\right)^{-1}\left(\left(1+\frac{n_{\mathrm{i}}}{n_{\mathrm{H}}}\right) \frac{v_{\text {in }}}{k V_{\mathrm{a}}}\right)^{2}\right)^{-1}$

was taken into account whereas the term $\Gamma_{\mathrm{nl}}$ that describes nonlinear dissipation was omitted. Here $v_{\mathrm{in}}=n_{\mathrm{H}}\left\langle v_{\mathrm{th}} \sigma\right\rangle \approx$ $8.4 \times 10^{-9}\left(T / 10^{4} \mathrm{~K}\right)^{0.4}\left(n_{\mathrm{H}} / 1 \mathrm{~cm}^{-3}\right) \mathrm{s}^{-1}$ for the temperature $T \sim 10^{2}-10^{5} \mathrm{~K}$ is the frequency of ion-neutral collisions with cross section $\sigma$ averaged over the velocity distribution of thermal particles, $n_{\mathrm{H}}$ is the number density of neutral hydrogen. 
(Note a slight correction of the "collisional damping" curve in Fig. 1 comparing to corresponding Fig. 2 in Paper I.) The maximum energy of protons accelerated by $\mathrm{SN}$ shocks at the early Sedov stage is close to $3 \times 10^{14} \mathrm{eV}$, which exceeds the Bohm limit calculated for the interstellar magnetic field value by one order of magnitude. The maximum energy decreases to about $10^{10} \mathrm{eV}$ at the end of the Sedov stage, which is much less than the Bohm limit calculated for the interstellar magnetic field value. In particular, the particle energy is less than $10^{13} \mathrm{eV}$ at $t>3 \times 10^{3} \mathrm{yr}$ and this may explain the absence of a TeV $\gamma$-ray signal from many SNRs (Buckley et al. 1998; Aharonian et al. 2002) where the gamma-rays could in principle be produced through $\pi^{0}$ decays if sufficiently energetic cosmic rays were present.

With the extreme choice of parameters of the rapidly expanding young SNR envelope, it was found (Bell \& Lucek 2001, Paper I) that the maximum particle energy may reach ultra high energies. The estimate of the highest particle energy according to Paper $\mathrm{I}$ is $E_{\max } \approx 2 \times 10^{17} Z\left(u_{\mathrm{sh}} / 3 \times\right.$ $\left.10^{4} \mathrm{~km} \mathrm{~s}^{-1}\right)^{2}(\varkappa / 0.1) \xi_{\mathrm{cr}} M_{\mathrm{ej}}^{1 / 3} n^{1 / 6} \mathrm{eV}$ at the end of a free expansion stage which precedes the Sedov stage (here $M_{\mathrm{ej}}$ is the mass of the ejecta in $M_{\odot}$ ). We shall see below that this promising estimate is in some sense devalued by the results of the calculations of the particle flux - the flux turns out to be low at the highest energies which can be achieved in the process of acceleration.

\section{Evolution of SNR shocks}

A typical source of galactic cosmic rays is most probably associated with the core collapse supernova, type II SNe, that is the final stage of evolution for stars more massive than about 8 solar masses while on the main sequence. Before the explosion, the massive star goes through the Main Sequence O-star stage, the Red Supergiant stage, and, for the most massive progenitors ( $>20 M_{\odot}$ ), which give rise to the rare type $\mathrm{Ib} / \mathrm{c} \mathrm{SNe}$, through the Wolf-Rayet stage. The fast wind of a massive progenitor star on the main sequence produces a big bubble of hot rarefied gas with a temperature of about $10^{6} \mathrm{~K}$ in the surrounding interstellar medium, see Weaver et al. (1977), Lozinskaya (1992). The typical type II SN goes through the Red Super Giant phase before the explosion and this process is accompanied by the flow of a low-velocity dense wind. Thus, immediately after the supernova burst, the shock propagates through the wind of a Red Super Giant star, then through the hot bubble, and finally it enters the interstellar medium. Our calculations will be done for an ejecta mass $M_{\mathrm{ej}}=1 M_{\odot}$ (the solar mass). The spherically symmetric distribution of gas density in the stellar wind is $n_{\mathrm{w}}=\dot{M} /\left(4 \pi m_{\mathrm{a}} u_{\mathrm{w}} r^{2}\right)$, where $\dot{M}=10^{-5} \dot{M}_{-5}$ (solar mass) $/ \mathrm{yr}$ is the mass loss rate, $m_{\mathrm{a}}=1.4 m$ is the mean interstellar atom mass per hydrogen nucleus, the wind velocity $u_{\mathrm{w}}=10^{6} u_{\mathrm{w}, 6} \mathrm{~cm} / \mathrm{s}$. The magnetic field in the stellar wind has the shape of a Parker spiral similar to the interplanetary magnetic field (Parker 1958). At the relatively large distances from the surface of the star that are of interest here the magnetic field has a predominately azimuthal structure and its value is $B_{0}=B_{*} r_{*}^{2} \Omega \sin \theta /\left(u_{\mathrm{w}} r\right)$ where $B_{*}$ is the surface magnetic field strength at the star radius $r_{*}, \Omega$ is the angular velocity of star rotation, and $\theta$ is the polar angle. Hence $B_{0}(r) r=2 \times 10^{13} u_{\mathrm{w}, 6}^{-1} \sin \theta \mathrm{G} \times \mathrm{cm}$ at $B_{*}=1 \mathrm{G}$, $r_{*}=3 \times 10^{13} \mathrm{~cm}, \Omega=3 \times 10^{-8} \mathrm{~s}^{-1}$ that gives $B_{0} \approx 6 \mu \mathrm{G}$ at the distance $r=1 \mathrm{pc}$ from the star.

Below we shall also use the following set of parameters of the medium surrounding the type II SN: the radius of the spherical Red Super Giant wind $R_{\mathrm{w}}=2 \mathrm{pc}$, the star mass loss $\dot{M}_{-5}=1$, and the wind velocity $u_{\mathrm{w}, 6}=1$. In addition, the radius of the spherical bubble of hot gas $R_{\mathrm{b}}=60 \mathrm{pc}$, the gas density in the bubble $n_{\mathrm{b}}=1.5 \times 10^{-2} \mathrm{~cm}^{-3}$, the magnetic field $B_{\mathrm{b}}=5 \mu \mathrm{G}$. The gas density in the undisturbed interstellar medium around the bubble is assumed to be equal to $n_{0}=1 \mathrm{~cm}^{-3}$ (physically, the value of $n_{0}$ determines $n_{\mathrm{b}}$, see Weaver et al. 1977). The hot bubble is separated by a dense thin shell from the interstellar gas. The accepted parameters are close to those assumed by Berezhko \& Völk (2000) in their analysis of gamma-ray production in SNRs. A lengthy discussion and additional references can be found there.

A considerable fraction of cosmic rays is probably accelerated in type Ia SNe (their explosion rate in the Galaxy is about $1 / 4$ of that of type II SN). These supernovae are caused by the thermonuclear explosions of compact white dwarfs following mass accretion. The characteristic masses of the progenitor star and ejecta are 1.4 solar mass. The progenitor stars do not appear to have an observable amount of mass loss nor do they emit ionizing radiation that could modify the ambient medium. We assume that the SNR shock goes through the uniform weakly ionized interstellar medium with density $1 \mathrm{~cm}^{-3}$, and magnetic field $7 \mu \mathrm{G}$.

It is instructive to consider the asymptotic regimes of the propagation of SNR shock - the ejecta dominated stage and the adiabatic stage.

The adiabatic regime was mentioned earlier (see the Sedov solution (11) for the shock moving in a gas with constant density), and it refers to the stage of SNR evolution when the mass of swept-up gas significantly exceeds the mass of ejecta. This condition is fulfilled in the case of a medium with constant density at $R_{\mathrm{sh}}>R_{0}=\left(3 M_{\mathrm{ej}} / 4 \pi m_{\mathrm{a}} n_{0}\right)^{1 / 3}=1.9\left(M_{\mathrm{ej}} / M_{\odot} n_{0}\right)^{1 / 3}$ pc, $t_{0}>R / u_{0} \approx 190 n_{0}^{-1 / 3} \mathrm{yr}$, where $u_{0} \sim 10^{9} \mathrm{~cm} / \mathrm{s}$ is the initial velocity of the ejecta. The adiabatic regime for the SNR shock moving through the progenitor star wind is described by the equations (at $u_{\mathrm{sh}} \gg u_{\mathrm{w}}$ ):

$$
\begin{aligned}
& R_{\mathrm{sh}}=7.9\left(\frac{\mathcal{E}_{51} u_{\mathrm{w}, 6}}{\dot{M}_{-5}}\right)^{1 / 3} t_{\mathrm{Kyr}}^{2 / 3} \mathrm{pc}, \\
& u_{\mathrm{sh}}=5.2 \times 10^{3}\left(\frac{\mathcal{E}_{51} u_{\mathrm{w}, 6}}{\dot{M}_{-5}}\right)^{1 / 3} t_{\mathrm{Kyr}}^{-1 / 3} \mathrm{~km} \mathrm{~s}^{-1},
\end{aligned}
$$

see Ostriker \& McKee (1988). As in Eq. (11), we assume that the ultrarelativistic gas of cosmic rays mainly determines the pressure behind the shock. Equation (13) is valid when the mass of swept-up gas is relatively large and $R_{\mathrm{sh}}>R_{0}=$ $M_{e j} u_{\mathrm{w}} / \dot{M} \approx 1\left(M_{\mathrm{ej}} / M_{\odot}\right) u_{\mathrm{w}, 6} / \dot{M}_{-5} \mathrm{pc}$.

The quantity $\rho u_{\mathrm{sh}}^{2} R_{\mathrm{sh}}^{3}=K \mathcal{E}$ is conserved for the adiabatic shocks considered. The constant $K \approx 0.16$ for solution (11), and $K \approx 0.34$ for solution (13). In the general case of a powerlaw gas distribution $\rho=\rho_{0}(r) r^{-s}, s<5$, the adiabatic shock evolution is described by the equations $R_{\mathrm{sh}}=\left(\eta(s) \mathcal{E} / \rho_{0}\right)^{\frac{1}{5-s}} t^{\frac{2}{5-s}}$, and $u_{s h}=\frac{2}{5-s}\left(\eta(s) \mathcal{E} / \rho_{0}\right)^{\frac{1}{5-s}} t^{-\frac{3-s}{5-s}}$, where $\eta$ is constant at fixed $s$, 
which gives the general formula $K=\frac{4 \eta(s)}{(5-s)^{2}}$ (the values of $\eta(s)$ were given by Ostriker \& McKee 1988).

The ejecta-dominated stage precedes the adiabatic one. As long as the mass of the ejecta is large compared to the swept-up mass, the blast wave is moving with relatively weak deceleration. At this stage, shortly after the explosion, the structure of the rapidly expanding envelope of the presupernova star is important for the shock evolution. Actually, the blast wave consists of two shocks, the forward shock and the reverse shock, with the contact discontinuity surface between them. This surface separates the shocked wind or interstellar gas downstream of the forward shock from the shocked envelope gas that fills the downstream region of the reverse shock. The reverse shock lags behind the forward shock and enters the dense internal part of the exploding star by the time of the beginning of the Sedov stage. Though as an approximation for very young SNRs it cannot be well justified, we ignore below the cosmic ray acceleration at the reverse shock compared to the forward shock and use the notation $R_{\mathrm{sh}}$ for the radius of forward shock (Berezinsky \& Ptuskin 1989 considered the cosmic ray acceleration by both shocks; see also Yoshida \& Yanagita 2001). The outer part of the star that freely expands after the SN explosion has a power law density profile $\rho_{\mathrm{s}} \propto r^{-k}$, see e.g. Chevalier \& Liang (1989). The value of $k$ typically lies between 6 and 14. The value $k \approx 7$ is characteristic of the $\mathrm{SNe}$ type Ia, and $k \approx 10$ is typical of the SNe type II. A self similar solution for the blast wave at the ejecta-dominated stage was found by Chevalier (1982) and Nadyozhin $(1981,1985)$. They showed that at an age larger than about one week, the evolution of the shock at the ejecta-dominated stage can be approximately described by the power-law dependence $R_{\text {sh }} \propto t^{\lambda}$ where the expansion parameter $\lambda=\frac{k-3}{k}$ for the explosion in the uniform medium, and $\lambda=\frac{k-3}{k-2}$ for the explosion in the wind of a presupernova star (for $k>5$ ejecta). In particular, using the results of the two papers mentioned above, one can obtain the following equations

$R_{\mathrm{sh}}=5.3\left(\frac{\mathcal{E}_{51}^{2} M_{\odot}}{n_{0} M_{\mathrm{ej}}}\right)^{1 / 7} t_{\mathrm{Kyr}}^{4 / 7} \mathrm{pc}$,

$u_{\mathrm{sh}}=2.7 \times 10^{3}\left(\frac{\mathcal{E}_{51}^{2} M_{\odot}}{n_{0} M_{\mathrm{ej}}}\right)^{1 / 7} t_{\mathrm{Kyr}}^{-3 / 7} \mathrm{~km} \mathrm{~s}^{-1}$

for a type Ia SN explosion in an uniform interstellar medium at $k=7$;

$R_{\mathrm{sh}}=7.7\left(\frac{\mathcal{E}_{51}^{7 / 2} u_{\mathrm{w}, 6} M_{\odot}^{5 / 2}}{\dot{M}_{-5} M_{\mathrm{ej}}^{5 / 2}}\right)^{1 / 8} t_{\mathrm{Kyr}}^{7 / 8} \mathrm{pc}$,

$u_{\mathrm{sh}}=6.6 \times 10^{3}\left(\frac{\mathcal{E}_{51}^{7 / 2} u_{\mathrm{w}, 6} M_{\odot}^{5 / 2}}{\dot{M}_{-5} M_{\mathrm{ej}}^{5 / 2}}\right)^{1 / 8} t_{\mathrm{Kyr}}^{-1 / 8} \mathrm{~km} \mathrm{~s}^{-1}$

for a type II SN explosion in the wind of a presupernova star at $k=10$.

Following the approach of Truelove \& McKee (1999), one can describe the shock produced by a type Ia SN using the continuous solution which coincides with the ejecta-dominated Eq. (14) until the moment $t_{0}=$ $260\left(M_{\mathrm{ej}} / 1.4 M_{\odot}\right)^{5 / 6} \mathcal{E}_{51}^{-1 / 2} n_{0}^{-1 / 3} \mathrm{yr}$, and is given by the equations

$$
\begin{aligned}
R_{\mathrm{sh}}= & 4.3\left(\mathcal{E}_{51} / n_{0}\right)^{1 / 5} t_{\mathrm{Kyr}}^{2 / 5} \\
& \times\left(1-\frac{0.06\left(M_{\mathrm{ej}} / M_{\odot}\right)^{5 / 6}}{\mathcal{E}_{51}^{1 / 2} n_{0}^{1 / 3} t_{\mathrm{Kyr}}}\right)^{2 / 5} \mathrm{pc}, \\
u_{\mathrm{sh}}= & 1.7 \times 10^{3}\left(\mathcal{E}_{51} / n_{0}\right)^{1 / 5} t_{\mathrm{Kyr}}^{-3 / 5} \\
& \times\left(1-\frac{0.06\left(M_{\mathrm{ej}} / M_{\odot}\right)^{5 / 6}}{\mathcal{E}_{51}^{1 / 2} n_{0}^{1 / 3} t_{\mathrm{Kyr}}}\right)^{-3 / 5} \mathrm{~km} \mathrm{~s}^{-1}
\end{aligned}
$$

at a later time $t>t_{0}$. It is evident from Eq. (16) that the adiabatic approximation (11) holds at $t \gg t_{0}$.

The evolution of a type II SN shock first follows the ejectadominated solution (15) in a presupernova wind and then, while still moving in the wind, it enters the adiabatic regime at the distance $r \sim 1 \mathrm{pc}$. The subsequent evolution proceeds in the medium with a complicated structure described above for a type II SN. The fairly accurate solution for the SNR evolution during this period can be obtained in the "thin-shell" approximation, e.g. Ostriker \& McKee (1988), Bisnovatyi-Kogan \& Silich (1995). Using this approximation for the strong shock and assuming a spherically symmetric distribution of the circumstellar gas density $\rho(r)$, we come to the following equations where the shock velocity $u_{\mathrm{sh}}$ and the SNR age $t$ are parameterized as functions of the shock radius $R_{\mathrm{sh}}$ (see Appendix for the derivation of these equations):

$$
\begin{aligned}
u_{\mathrm{sh}}\left(R_{\mathrm{sh}}\right)= & \frac{\gamma_{\mathrm{ad}}+1}{2}\left[\frac{12\left(\gamma_{\mathrm{ad}}-1\right) \mathcal{E}}{\left(\gamma_{\mathrm{ad}}+1\right) M^{2}\left(R_{\mathrm{sh}}\right) R_{\mathrm{sh}}^{6\left(\gamma_{\mathrm{ad}}-1\right) /\left(\gamma_{\mathrm{ad}}+1\right)}}\right. \\
& \left.\times \int_{0}^{R \mathrm{sh}} \mathrm{d} r r^{6\left(\frac{\gamma_{\mathrm{ad}}-1}{\gamma_{\mathrm{ad}}+1}\right)-1} M(r)\right]^{1 / 2} \\
t\left(R_{\mathrm{sh}}\right)= & \int_{0}^{R_{\mathrm{sh}}} \frac{\mathrm{d} r}{u_{\mathrm{sh}}(r)}
\end{aligned}
$$

where $\gamma_{\mathrm{ad}}$ is the adiabatic index $\left(\gamma_{\mathrm{ad}}=4 / 3\right.$ if the pressure downstream of the shock is determined by the relativistic particles), and $M(R)=M_{e j}+4 \pi \int_{0}^{R} \mathrm{~d} r r^{2} \rho(r)$ is the mass of the swept-up gas. The self-similar solution by Chevalier and by Nadyozhin is not explicitly reproduced by Eqs. (17). The solutions (15) and (17) are fitted together at the transition from the ejecta-dominated regime to the adiabatic regime (at $r \sim 0.3 \mathrm{pc}$ ) in our numerical simulations of cosmic ray acceleration in the type II SNRs described below.

It is worth noting that the energy loss of SNRs in the form of escaping cosmic rays is not taken into account in the solutions for shock evolution that were described in this section. In fact the shock evolution is only approximately adiabatic.

\section{Average spectrum of cosmic rays injected in the interstellar medium}

At a given SNR age $t$, the cosmic rays are accelerated up to a maximum momentum $p_{\max }(t)$. Also, particles with $p>$ $p_{\max }(t)$ cannot be confined in the precursor of the shock even if they were accelerated earlier. Thus particles accelerated to 
the maximum energy escape from a SNR (see also Berezhko \& Krymsky 1988). Let us estimate the flux of these run-away particles. We consider the simplified approach for the maximum energy of the accelerated particles and take the dependence of diffusion on momentum in the following simplified form:

$$
\begin{aligned}
& D(p)=D_{0} \ll R u_{\mathrm{sh}}, p \leqslant p_{\max }(t), \\
& D(p)=D_{\mathrm{m}} \gg R u_{\mathrm{sh}}, p>p_{\max }(t) .
\end{aligned}
$$

The spectrum of the accelerated particles in this case has a very steep cut-off at $p>p_{\max }$ (cf. Eq. (1)) and the spectrum of run-away particles beyond $p_{\max }$ can be approximated by a $\delta$ function. To find the equation for these particles, let us integrate the equation for the momentum distribution function of cosmic rays

$$
\frac{\partial f}{\partial t}-\nabla D \nabla f+\boldsymbol{u} \nabla f-\frac{\nabla \boldsymbol{u}}{3} p \frac{\partial f}{\partial p}=0
$$

on momentum $p$ from $p_{\max }$ to $p_{\max }+\Delta p$, where $\Delta p \ll p_{\max }$ and larger than the width of the run-away particle spectrum. Denoting $G=\int_{p_{\max }}^{p_{\max }+\Delta p} f \mathrm{~d} p$ one obtains from Eq. (19):

$$
\begin{aligned}
& \frac{\partial G}{\partial t}+\boldsymbol{u} \nabla G= \\
& \nabla D_{\mathrm{m}} \nabla G-\frac{\partial p_{\max }}{\partial t} f\left(p_{\max }-0\right)-\frac{\nabla \boldsymbol{u}}{3} p_{\max } f\left(p_{\max }-0\right) .
\end{aligned}
$$

Since the diffusion coefficient of the run-away particles is large, the advection terms play no role in this equation and the last two terms can be considered as a source of the particles. The total source of run-away particles is given by the volume integral of these terms. As a result, the source spectrum of the run-away particles has the form

$$
\begin{aligned}
q(p, t)= & -\delta\left(p-p_{\max }\right) \\
& \times \int \mathrm{d}^{3} r\left(\frac{\partial p_{\max }}{\partial t}+\frac{\nabla \boldsymbol{u}}{3} p_{\max }\right) f\left(p_{\max }-0, \boldsymbol{r}\right) .
\end{aligned}
$$

The integration here is performed over the domain where the integrand is negative. The integral $4 \pi \int \mathrm{d} p p^{2} q(p, t)$ has dimensions number of particles per unit time.

Below we consider the case of a spherically symmetric SN shock with linear velocity profile at $r<R_{\mathrm{sh}}$ :

$u=\left(1-\frac{1}{\sigma}\right) u_{\mathrm{sh}}(t) r / R_{\mathrm{sh}}(t)$,

where $\sigma$ is the total shock compression ratio. It includes a thermal subshock and a cosmic ray precursor. The linear velocity profile (22) is a good approximation of Sedov's solution and it can be considered as a very approximate one at the ejectadominated stage. Since the shock is partially modified in the presence of cosmic rays, we should not assume any relation between the shock compression ratio $\sigma$ and the spectral index of accelerated particles $4-a$ (recall that $4-a=3 \sigma /(\sigma-1)$ for unmodified shocks). We accept the value $\sigma=7$ in our calculations. The preshock at $r>R_{\mathrm{sh}}$ is created by the cosmic ray pressure gradient. Its width is small in comparison with the shock radius under the conditions given by Eq. (18) and the plane shock approximation can be used. Since the cosmic ray pressure dominates the gas pressure in the precursor region, its gradient is proportional to the velocity gradient $\partial P_{\mathrm{cr}} / \partial r=\rho u_{\mathrm{sh}} \partial u / \partial r$, where $\rho$ is the density of the circumstellar medium. We also use the assumption that the cosmic ray pressure at the shock is some fraction $\xi_{\mathrm{cr}}$ of the upstream momentum flux, see Eq. (1). Now assuming that $f\left(p_{\max }\right)$ is proportional to the cosmic ray pressure the expression (21) for the run-away particle source takes the form

$$
\begin{aligned}
q(p, t)= & 4 \pi \delta\left(p-p_{\max }\right)\left(\frac{1}{3}\left(1-\frac{1}{\sigma}-\frac{\xi_{\mathrm{cr}}}{2}\right) R^{2} u_{\mathrm{sh}} p f_{0}(p)\right. \\
& \left.-\int_{0}^{R} r^{2} \mathrm{~d} r f\left(p_{\max }, r\right)\left(\frac{\partial p_{\max }}{\partial t}+\frac{\sigma-1}{\sigma} p_{\max } \frac{u_{\mathrm{sh}}}{R}\right)\right) .
\end{aligned}
$$

The first term in this expression describes the particles which runs away from the shock front, and the second term describes the particles escaping from the shock interior. In principle, the turbulence downstream from the strong shock might be enhanced, which would result in a small cosmic ray diffusion coefficient. In this case the particles do not run away from the downstream and the second term in Eq. (23) should be omitted. If the turbulence downstream is maintained by the same process of cosmic ray streaming instability as in the upstream region, the downstream diffusion coefficient is comparable to the upstream diffusion coefficient for particles with $p \sim p_{\max }$. We shall further assume that particles can run away both from upstream and downstream of the shock. The uncertainty of the efficiency of the run-away process in the inner part of SNR does not qualitatively change the conclusion about the average source spectrum of cosmic rays calculated later in this section and shown in Fig. 2.

The distribution function of particles with $p \leqslant p_{\max }$ can be found using the solution of transport Eq. (19) at $r<R_{\text {sh }}$ with the boundary condition $f\left(p, r=R_{\mathrm{sh}}, t\right)=f_{0}$ by the method of characteristics. This gives:

$$
\begin{aligned}
q(p, t)= & 4 \pi \delta\left(p-p_{\max }\right)\left[\frac{1}{3}\left(1-\frac{1}{\sigma}-\frac{\xi_{\mathrm{cr}}}{2}\right) R^{2} u_{\mathrm{sh}} p f_{0}(p)\right. \\
& +\left(-\frac{\partial p_{\max }}{\partial t}-\frac{\sigma-1}{\sigma} p_{\max } \frac{u_{\mathrm{sh}}}{R}\right) \int_{0}^{t} \frac{\mathrm{d} t^{\prime}}{\sigma} R^{2}\left(t^{\prime}\right) u_{\mathrm{sh}}\left(t^{\prime}\right) \\
& \left.\times f_{0}\left(p\left(\frac{R(t)}{R\left(t^{\prime}\right)}\right)^{1-\frac{1}{\sigma}}, t^{\prime}\right)\left(\frac{R(t)}{R\left(t^{\prime}\right)}\right)^{3-\frac{3}{\sigma}}\right] .
\end{aligned}
$$

The expression in brackets in front of the integral in Eq. (24) should be positive, which means that adiabatically the particles lose energy more slowly than the maximum energy decreases. For the opposite sign, the adiabatic losses of particles are faster than the decrease of maximum energy and the particles do not run away from downstream of the shock. They can run away later if at that time the decrease of the maximum momentum is faster.

The average source power $Q(p)$ of run-away cosmic rays per unit volume in the galactic disk is obtained by integrating $q$ with respect to $t$ and by averaging over many SN explosions: $Q(p)=v_{\mathrm{sn}} \int_{\min }^{\max } \mathrm{d} t q(p, t)$, where $v_{\mathrm{sn}}$ is the average frequency of SN explosions per unit volume of the galactic disk. Changing 
the variable of integration from $t$ to $R_{\mathrm{sh}}\left(\mathrm{d} R_{\mathrm{sh}}=u_{\mathrm{sh}} \mathrm{d} t\right)$ one can derive the following equation:

$$
\begin{aligned}
Q(p)= & \frac{3 a \xi_{\mathrm{cr}} v_{\mathrm{sn}}}{c p^{4}\left|\frac{\mathrm{d} \ln \left(\mathrm{p}_{\max }\right)}{\mathrm{d}(R)}\right|}\left[\frac{\left(1-\frac{1}{\sigma}-\frac{\xi_{\mathrm{cr}}}{2}\right) \rho(R) u_{\mathrm{sh}}^{2}(R) R^{3}}{3\left(1-\frac{1}{1+a}\left(\frac{m c}{p_{\max }(R)}\right)^{a}\right)}\right. \\
& +\left(\frac{1}{\sigma}-1-\frac{\mathrm{d} \ln \left(p_{\max }\right)}{\mathrm{d} \ln (R)}\right) \int_{R_{\min }}^{R} \frac{\mathrm{d} R^{\prime}}{\sigma} \frac{\rho\left(R^{\prime}\right) u_{\mathrm{sh}}^{2}\left(R^{\prime}\right) R^{\prime 2}}{\left(1-\frac{1}{1+a}\left(\frac{m c}{p_{\max }\left(R^{\prime}\right)}\right)^{a}\right)} \\
& \times H\left(p_{\max }\left(R^{\prime}\right)-p\left(\frac{R}{R^{\prime}}\right)^{1-\frac{1}{\sigma}}\right) \\
& \left.\times\left(\frac{p}{p_{\max }\left(R^{\prime}\right)}\right)^{a}\left(\frac{R}{R^{\prime}}\right)^{(a-1)(\sigma-1) / \sigma}\right]_{R=R_{\mathrm{m}}(p)} .
\end{aligned}
$$

Here Eq. (1) for $f_{0}$ with the approximation $\varphi(p) \approx$ $a^{-1}(p / m c)^{a}-a^{-1}(1+a)^{-1}$ is used, and the condition $\xi_{\mathrm{cr}}=$ const. is assumed. The function $R_{\mathrm{m}}(p)$ in Eq. (25) is defined by the equation $p_{\max }\left(R_{\mathrm{sh}}=R_{\mathrm{m}}(p)\right)=p$. If the last equation has multiple solutions at a given $p$, the summation on all these solutions should be performed in Eq. (25). The physical meaning of $R_{\mathrm{m}}(p)$ is as follows: it is the value of the shock radius when the maximum momentum of accelerating particles is equal to $p$. The second term in the r.h.s. of Eq. (25) should be omitted if the expression in parentheses in front of the integral is negative.

Let us assume that the maximum momentum is a powerlaw function of the shock radius, $p_{\max } \propto R^{-\delta}$, the particles are ultrarelativistic, $p \gg m c$, and the compression ratio is constant, $\sigma=$ const. The remarkable feature of Eq. (25) is then that the expression in square brackets does not depend on momentum at the adiabatic stage of SNR shock propagation in the medium with a power-law distribution of the gas density, because $\rho u_{\mathrm{sh}}^{2} R_{\mathrm{sh}}^{3}=K \mathcal{E}, K=$ const. in this case, see Sect. 3. The average cosmic ray source power is now given by the simple equation

$$
\begin{aligned}
Q(p)= & \frac{3 K a \xi_{\mathrm{cr}} \nu_{\mathrm{sn}} \mathcal{E}}{c p^{4}}\left[\frac{1}{3 \delta}\left(1-\frac{1}{\sigma}-\frac{\xi_{\mathrm{cr}}}{2}\right)\right. \\
+ & \left.\frac{1}{\sigma} \frac{1-\frac{\sigma-1}{\sigma \delta}}{1-\frac{1}{\sigma}+a\left(\delta-1+\frac{1}{\sigma}\right)}\right] .
\end{aligned}
$$

Here the factors $\delta$, and $\left(1-\frac{\sigma-1}{\sigma \delta}\right)$ should be positive. The first term in the square brackets describes the particles which run away from the shock and the second term describes the particles which run away from the SNR interior. Consequently, while in the adiabatic regime, the SNR shock during its evolution produces run-away particles with the universal power-law overall spectrum $Q(p) \propto p^{-4}$, whereas the instantaneous spectrum at the shock is more flat and not universal (see Eq. (1)) and the instantaneous spectrum of run-away particles has a deltafunction form (see Eq. (24)).

The total source power of ultrarelativistic particles calculated with the use of Eq. (26) is $W=$ $4 \pi c \int \mathrm{d} p p^{3} Q(p)=C \xi_{\mathrm{cr}} v_{\mathrm{sn}} \mathcal{E} \ln \left(p_{\max 2} / p_{\max 1}\right)$, where $C=12 \pi K a\left(\frac{1}{3 \delta}\left(1-\frac{1}{\sigma}-\frac{\xi_{\mathrm{cr}}}{2}\right)+\frac{1}{\sigma} \frac{1-\frac{\sigma-1}{\sigma \delta}}{1-\frac{1}{\sigma}+a\left(\delta-1+\frac{1}{\sigma}\right)}\right) ; \quad p_{\max 2}$ and $p_{\max 1}$ are the maximum momenta of accelerated particles at the beginning and at the end of the adiabatic stage respectively, thus typically $\ln \left(p_{\max 2} / p_{\max 1}\right) \approx 10$. This leads to the estimate $W \approx 0.5\left(\xi_{\mathrm{cr}} / 0.5\right) \nu_{\mathrm{sn}} \mathcal{E}$ for the shock moving in the uniform interstellar medium. Hence a considerable part of the total available mechanical energy of $\mathrm{SN}$ explosion $v_{\mathrm{sn}} \mathcal{E}$ goes into cosmic rays at $\xi_{\text {cr }} \sim 0.5$. As is well known, the source spectrum $\propto p^{-4}$ or slightly steeper, and an efficiency of cosmic ray acceleration at the level $10-30 \%$ are needed to fit the cosmic ray data below the knee in the cosmic ray spectrum at about $4 \times 10^{15} \mathrm{eV}$ in the empirical model of cosmic ray origin (e.g. Ptuskin 2001, see also discussion below).

At the ejecta-dominated stage which precedes the adiabatic stage, the average spectrum of the run-away particles is different from $p^{-4}$. Let us consider the general case and assume that $\rho \propto r^{-s}, R_{\mathrm{sh}} \propto t^{\lambda}$ and hence $u_{\mathrm{sh}} \propto t^{\lambda-1}$. The maximum momentum of accelerated particles in the high velocity limit (7) has the scaling $p_{\max } \propto u_{\mathrm{sh}}^{2} R_{\mathrm{sh}} \rho^{1 / 2} \propto t^{\lambda\left(3-\frac{s}{2}\right)-2} \propto R_{\mathrm{sh}}^{3-\frac{s}{2}-\frac{2}{\lambda}}$, so that $R_{\mathrm{m}}(p) \propto p^{\frac{1}{3-\frac{s}{2}-\frac{2}{\lambda}}}$. Now Eq. (25) at $\lambda<4 /(6-s)$ gives the following shape of the average spectrum of run-away particles:

$$
Q(p) \propto p^{-4-\frac{\lambda(5-s)-2}{2-\lambda\left(3-\frac{s}{2}\right)}}
$$

A characteristic of the adiabatic regime is the relation $\lambda=\frac{2}{5-s}$ and therefore Eq. (27) gives $Q(p) \propto p^{-4}$ in agreement with Eq. (26). The Chevalier - Nadyozhin solution for the ejectadominated stage has $\lambda=\frac{k-3}{k-s}$. With the set of parameters excepted in Section 3, we have then $Q(p) \propto p^{-6.5}$ for the acceleration at the shock produced by a type II SN in the presupernova star wind $(s=2, k=10, \lambda=7 / 8)$, and $Q(p) \propto p^{-7}$ for the acceleration at the shock produced by a type Ia SN in a uniform interstellar medium $(s=0, k=7, \lambda=4 / 7)$. Thus the cosmic rays accelerated at the ejecta-dominated stage have higher energies than at the later adiabatic stage but the average energy spectrum of produced cosmic rays is rather steep at the "canonical" choice of presupernova star parameters.

The results of our numerical calculations of the average spectra for type II and type Ia SNe are shown in Fig. 2. The parameter $x$ is equal to 0.1 in a high-velocity regime (7) and it is equal to 0.04 in a low-velocity regime (9).

The calculations for type II SN are based on Eqs. (5), (6), (15), (17) and (25). For the set of parameters accepted in the present paper, the type II supernovae are able to accelerate cosmic ray protons to a maximum energy of the order $4 \times 10^{16} \mathrm{eV}$ if the acceleration starts one week after the SN explosion when $u_{\mathrm{sh}} \approx 2.4 \times 10^{4} \mathrm{~km} \mathrm{~s}^{-1}$. The energy spectrum is close to $p^{-4}$ at energies less than about $6 \times 10^{15} \mathrm{eV}$ and it steepens above this energy. Thus the proton knee lies at about $6 \times 10^{15} \mathrm{eV}$, in good agreement with the observational data. The sharp dip in the average proton spectrum at $p / m c \sim 1 \times 10^{6}-3 \times 10^{6}$ is caused by the assumed abrupt change of the gas density at the boundary between the dense Red Super Giant wind and the low density bubble. We ran calculations up to the maximum shock radius $60 \mathrm{pc}$ (the corresponding SNR age is $9 \times 10^{4} \mathrm{yr}$ ) when the Mach number approaches 3 and the use of the particle spectrum (1) characteristic of the strongly modified shocks can no longer be justified. The protons are accelerated to about $20 \mathrm{GeV}$ at this moment. 


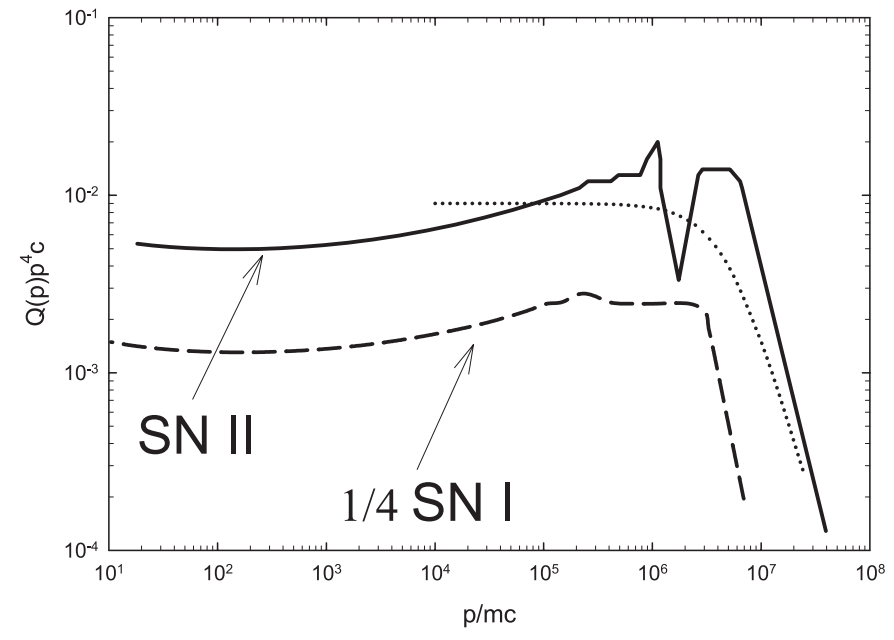

Fig. 2. The solid line shows the average source spectrum $Q(p) p^{4} c$ (given in units $\frac{\xi_{\mathrm{cr}}}{05} v_{\mathrm{sn}} \mathcal{E}$ per steradian) for protons released into the interstellar medium during SNR evolution after SNII explosion in the wind of a RSG progenitor star. The dashed line presents the case of a SNIa explosion in a uniform interstellar gas; the average source spectrum is multiplied by $1 / 4$. The dotted line shows the shape of the proton source spectrum used by Hörandel (2003) to fit the KASCADE data.

The calculations for type Ia $\mathrm{SN}$ in Fig. 2 are based on Eqs. (5), (6), (14), (16) and (25). The calculations were made for a shock radius range from 0.2 to $30 \mathrm{pc}$ (SNR age from $4 \mathrm{yr}$ to $1.3 \times 10^{5} \mathrm{yr}$ ). The shock velocity changes during this period from $2.8 \times 10^{4} \mathrm{~km} \mathrm{~s}^{-1}$ to $91 \mathrm{~km} \mathrm{~s}^{-1}$. The protons are accelerated from the maximum energy $7 \times 10^{15} \mathrm{eV}$ to about $10 \mathrm{GeV}$. The approximate proton spectrum $p^{-4}$ extends to the knee at about $3 \times 10^{15} \mathrm{eV}$.

The average source spectrum produced by type Ia $\mathrm{SNe}$ is multiplied by $1 / 4$ in Fig. 2, which corresponds to their relative explosion rate and hence reflects the relative contribution of this type of supernovae to the total production of cosmic rays in the Galactic disk as compared to the contribution of type II SNe.

\section{Consistency with cosmic ray data and discussion}

The spectrum of high-energy cosmic rays in the Galaxy is of the form $f \propto p^{-\gamma}, \gamma=\gamma_{\mathrm{s}}+b$ under the steady state conditions in which the action of cosmic ray sources (with source power $Q \propto p^{-\gamma_{\mathrm{s}}}$ ) is balanced by the escape of energetic particles from the Galaxy (with escape time $T \propto p^{-b}$ ). The all-particle spectrum of cosmic rays observed at the Earth is close to $f \propto p^{-4.7}$ at energies $E \gtrsim 10 \mathrm{GeV} /$ nucleon with a characteristic transition (the knee, Kulikov \& Khristiansen 1958) ranging over less than one decade in the vicinity of $4 \times 10^{15} \mathrm{eV}$ to the another powerlaw $f \propto p^{-5.1}$. The latter extends to about $5 \times 10^{17} \mathrm{eV}$ where the second knee with a break $\delta \gamma \sim 0.3$ is seen in the cosmic ray spectrum, see (Hörandel 2003) for a review. This structure is usually associated with a severe decrease of the efficiency of cosmic ray acceleration and/or confinement in the Galaxy.
The extragalactic component of cosmic rays probably dominates at $E \gtrsim 3 \times 10^{18} \mathrm{eV}$ (Gaisser et al. 1993). In the alternative interpretation (Berezinsky et al. 2004), the Galactic component falls steeply (with $\gamma \sim 6$ ) at $E \gtrsim 10^{17} \mathrm{eV}$ and the extragalactic component dominates from energy $\sim 3 \times 10^{17} \mathrm{eV}$ and above.

The exponent $b=0.3 \div 0.7$ was obtained from the data on the abundance of secondary nuclei at energies $10^{9}$ to $10^{11} \mathrm{eV} /$ nucleon. The secondary nuclei are produced in cosmic rays in the course of nuclear fragmentation of more heavier primary nuclei moving through the interstellar gas. The uncertainty in the value of $b$ is mainly due to the choice of specific model of cosmic ray transport in the Galaxy, see Ptuskin (2001). It follows that the source exponent below the first knee lies in the range $\gamma_{\mathrm{s}}=4.0 \ldots 4.4$. The value $\gamma_{\mathrm{s}} \approx 4.0$ for the average source spectrum was obtained above in the consideration of particle acceleration by SNR shocks during their adiabatic evolution (though smaller $b \sim 0.3$ and consequently larger $\gamma_{\mathrm{s}} \sim 4.4$ would be more favorable for the explanation of the high isotropy of cosmic rays observed at $10^{12}$ to $\left.10^{14} \mathrm{eV}\right)$. According to the results of Sect. 4 the calculated average source spectrum $p^{-4}$ for protons accelerated by a "typical" type II SNe extends up to about $6 \times 10^{15} \mathrm{eV}$, which coincides with the observed position of the knee $\sim 4 \times 10^{15} \mathrm{eV}$ within the accuracy of our analysis. The knee position at $3-5 \mathrm{PeV}$ was determined in the recent KASCADE experiment (Ulrich et al. 2003). The scaling of the knee position in our model is $p_{\text {knee }} \propto Z \varkappa \xi_{\text {cr }} \mathcal{E} \dot{M}^{1 / 2} M_{\mathrm{ej}}^{-1} u_{\mathrm{w}}^{-1}$ for explosion in the stellar wind and $p_{\text {knee }} \propto Z \varkappa \xi_{\text {cr }} \mathcal{E} M_{\mathrm{ej}}^{-2 / 3} n_{0}^{1 / 6}$ for explosion in the uniform interstellar medium.

As was recalled earlier, the diffusive shock acceleration at the strong nonmodified shock produces the spectrum $f \propto p^{-4}$. The back reaction of efficiently accelerating particles modifies the shock structure, which results in a flatter particle spectrum (see references at the beginning of Sect. 2 and Eq. (1) where $a \sim 0.5$ if the shock modification is very strong). However, the numerical simulation of acceleration by SNR shocks under the standard assumption of Bohm diffusion in the shock precursor (calculated for the interstellar magnetic field strength) and with efficient confinement of accelerated particles during the whole of the SNR evolution gives an overall source spectrum that is close to $p^{-4}$ (Berezhko et al. 1996). Berezhko \& Völk (2000) pointed out that the last result is in some sense accidental. The late stages of SNR evolution are important here since a relatively weak shock produces a steep particle spectrum, which has an effect on the overall spectrum. The situation is different in the model discussed in the present paper because the diffusion coefficient increases strongly with SNR age and the cosmic rays with energies larger than $10-30 \mathrm{GeV} /$ nucleon leave the supernova shell as run-away particles when the shock remains strong. The final average source spectrum of high-energy cosmic rays with energies larger than $10-30 \mathrm{GeV} / \mathrm{n}$ is close to $p^{-4}$ provided that the shock evolution is approximately adiabatic and the efficiency of particle acceleration $\xi_{\text {cr }}$ is roughly constant. The source spectrum of particles with energies less than $10-30 \mathrm{GeV} / \mathrm{n}$ may be steeper because they are accelerated by not very strong shocks. In this connection it should be noted that the source spectrum in the basic empirical model of cosmic ray propagation in the Galaxy is of the form $Q(p) p^{2} \propto p^{-2.4}$ at 
$E<30 \mathrm{GeV} / \mathrm{n}$, and $Q(p) p^{2} \propto p^{-2.15}$ at $E>30 \mathrm{GeV} / \mathrm{n}$, see Jones et al. (2001).

There are other important differences between the standard models of cosmic-ray acceleration and the one presented here. As noted before, our model of cosmic ray acceleration with a strong increase in time of the diffusion coefficient and corresponding decrease of maximum particle energy may naturally explain why the SNRs are generally not bright in very high energy gamma-rays at an age larger than a few thousand years. At this period of time, there are no particles with the energies needed to generate the very high energy gamma-rays in a SNR shell. Another problem is the contribution of gammaray emission from numerous unresolved SNRs with relatively flat spectra to the diffuse galactic background at very high energies. Working in the standard model Berezhko \& Völk (2003) took the maximum possible energy of protons accelerated in SNRs equal to $10^{14} \mathrm{eV}$, i.e. well below the knee position, and this made it possible not to exceed the upper limits on the diffuse gamma-ray emission at $4 \times 10^{11}$ to $10^{13} \mathrm{eV}$ obtained in the Whipple, HEGRA, and TIBET experiments. In the model considered in the present work even with efficient proton acceleration that may go beyond the knee, the expected gamma-ray emission from SNRs at $4 \times 10^{11} \mathrm{eV}$ is an order of magnitude smaller than in the model with Bohm diffusion. For a similar reason, the standard model compared with the present model predicts a larger ratio of fluxes of secondary and primary nuclei formed at very high energies through the reacceleration of secondaries by strong shocks and through the direct production of secondaries by primary nuclei with flat energy spectra inside SNRs, see Berezhko et al. (2003).

The interpretation of the energy spectrum beyond the knee in the present model is associated with the cosmic ray acceleration during the ejecta-dominated stage of SNR evolution when the protons gain energy that is larger by an order of magnitude than at the adiabatic stage but the number of particles involved in the shock acceleration is relatively small. The average source spectrum of accelerated particles is not universal at this stage. It is power law with an exponent $\gamma_{\mathrm{s}}$ whose value is very sensitive to the parameter $k$. The last is not well determined from the observations but the typical values accepted in our calculations were $k=10$ for a type II SN explosion in the wind of a Red Super Giant progenitor, and $k=7$ for a type Ia SN explosion in a uniform interstellar medium (see Chevalier \& Liang 1989) that give $\gamma_{\mathrm{s}}=6.5$ and $\gamma_{\mathrm{s}}=7$ respectively; see Sect. 4 . To illustrate the range of possible uncertainty, it is worth noting that the value $k=5.4$ was suggested for the type Ia SNe by Imshennik et al. (1981). This value of $k$ results in $\gamma_{\mathrm{s}}=4.3$ at the ejecta-dominated stage.

The breaks and cutoffs in the spectra of ions with different charges should occur at the same magnetic rigidity as for protons, i.e. at the same ratio $p / Z$ (or $E / Z$ for ultrarelativistic nuclei). The data of the KASCADE experiment (Ulrich et al. 2003) for the most abundant groups of nuclei (protons, helium, CNO group, and the iron group nuclei) are, in general, consistent with this concept. According to Hörandel (2003) a good fit to the observations is reached if an individual constituent ion spectrum has a gradual steepening by $\delta \gamma \sim 2$ at energy $4 \times 10^{15} \mathrm{Z} \mathrm{eV}$. Equation (27) shows that the value $\delta \gamma_{\mathrm{s}}=2 \mathrm{can}$ be obtained at $k=9, s=2$ (the $\mathrm{SN}$ explosion in the progenitor wind), or $k=6.6, s=0$ (the SN explosion in the uniform interstellar medium), which are not very different from our accepted "typical" values, see Fig. 2.

At present, the main problem of the data interpretation centers around the second knee in the cosmic ray spectrum. The natural assumption that all individual ions have only one knee at $\sim 4 \times 10^{15} \mathrm{Z} \mathrm{eV}$ and that the knee in the spectrum of iron $(Z=26)$ expected at about $10^{17} \mathrm{eV}$ explains the second knee in the all-particle spectrum does not agree with the observed position of the second knee at $5 \times 10^{17} \mathrm{eV}$. One way out was suggested by Hörandel (2003) who included all elements up to $Z=92$ into the consideration and assumed that $\gamma_{\mathrm{s}}$ decreases with $Z$ to raise the contribution of ultra heavy nuclei from Galactic sources to the cosmic ray flux at $\gtrsim 10^{17} \mathrm{eV}$. Of considerable promise is the approach by Sveshnikova (2003) who took into account the dispersion of parameters of SN explosions in her calculations of the knee position and the maximum particle energy. This leads to a widening of the energy interval between the two knees in the overall all-particle spectrum. This analysis should be supplemented by the account of different chemical compositions of the progenitor star winds that determine the composition of accelerated cosmic rays (Silberberg et al. 1991). We plan future work on this topic in the framework of the model developed in the present paper and Paper I. It should be noted that the model by Berezinsky et al. (2004) is quite consistent with the decreasing of the flux from Galactic sources above $10^{17} \mathrm{eV}$ since the conjunction with the intergalactic cosmic ray flux in their model occurs at relatively low energy.

There is also a very different scenario which assumes the strong reacceleration of cosmic rays above the knee by the collective effect of multiple SNR shocks in violent regions of the Galactic disk (Axford 1994; Bykov \& Toptygin 2001; Klepach et al. 2000) or Galactic wind (Völk \& Zirakashvili 2004).

Finally, it is worth noting that in principle the knee may arise not in the sources but in the process of cosmic ray propagation in the Galaxy, e.g. as a result of interplay between ordinary and Hall diffusion (Ptuskin et al. 1993; Roulet 2004). However, this explanation requires the existence of a powerlaw source spectrum which extends without essential breaks up to about $10^{18} \mathrm{eV}$ or even further.

\section{Conclusion}

The accounting for non-linear effects which accompany the cosmic ray streaming instability raises the maximum energy of accelerated particles in young SNRs above the standard Bohm limit by about two orders of magnitude. It also considerably reduces the maximum energy of particles that are present inside SNRs at the late Sedov stage if, as was assumed in our calculations in Sect. 4, the cosmic ray diffusion coefficient downstream of the shock is not much smaller than the diffusion coefficient in the cosmic ray precursor of the shock and the energetic particles with $p \sim p_{\max }$ run away from the SNR interior. In the present paper we studied the effect of a strong time dependence of the maximum particle momentum $p_{\max }(t)$ on the average spectrum of cosmic rays injected into 
interstellar space from many supernova remnants over their lifetime. The instantaneous cosmic ray spectrum at a strongly modified shock is flat $\left(f_{0} \propto p^{-4+a}, a>0\right.$, Eq. (1)) and the particle energy density is mainly determined by the particles with maximum momentum $p_{\max }(t)$. The instantaneous source spectrum of the run-away particles is close to a delta function $\left(q_{\mathrm{ra}}(t, p) \propto \delta\left(p_{\max }(t)-p\right)\right.$, Eq. (24)). At the same time, the assumption that a constant fraction $\xi_{c r}$ of incoming gas momentum flux goes into the cosmic ray pressure at the shock, and the fact that the supernova remnant evolution is adiabatic leads to an average source spectrum for ultrarelativistic particles from the ensemble of SNRs that is close to $Q_{\mathrm{ra}} \propto p^{-4}$ from energies 10-30 GeV/n up to the knee position in the observed cosmic ray spectrum independent of the value of $a$, (see Eq. (26) and Fig. 2). This source spectrum is consistent with the empirical model of cosmic ray propagation in the Galaxy. The acceleration at the preceding ejecta-dominated stage of SNR evolution provides the steep power-law tail in the particle distribution at higher energies up to $\sim 10^{18} \mathrm{eV}$ (if the iron nuclei dominate at these energies). The knee in the observed energy spectrum of cosmic rays at $\sim 4 \times 10^{15} \mathrm{eV}$ is explained in our model by the transition from the ejecta-dominate stage to the adiabatic stage of SNR shock evolution. In spite of the approximate character of our consideration, the suggested scenario of particle acceleration can explain the energy spectrum of Galactic cosmic rays.

Acknowledgements. The authors are grateful to Evgenij Berezhko and Heinz Völk for fruitful discussions. We thank the referee for valuable comments. This work was supported by an RFBR grant at IZMIRAN. VSP was also supported by a NASA grant during his visit to the University of Maryland where part of this work was carried out.

\section{Appendix A: Thin shell approximation}

The thin-shell approximation can be used when the swept-up gas is concentrated in a thin layer behind the shock. In particular, it is applied to the case of a spherical adiabatic shock, see Ostriker \& McKee (1988) and Bisnovatyi-Kogan \& Silich (1995) for details. The total mass of the gas shell involved in the motion and confined by the shock of radius $R_{\mathrm{sh}}$ in the spherically symmetrical case is

$$
M=M_{\mathrm{ej}}+4 \pi \int_{0}^{R_{\mathrm{sh}}} \mathrm{d} r r^{2} \rho(r)
$$

where $M_{\mathrm{ej}}$ is the ejected mass, $\rho$ is the density of ambient gas.

The equation of momentum conservation is

$$
\frac{\mathrm{d}(M u)}{\mathrm{d} t}=4 \pi R_{\mathrm{sh}}^{2}\left(P_{\mathrm{in}}-P\right)
$$

Here $u$ is the gas velocity behind the shock, $P_{\text {in }}$ is the pressure behind the shock, and $P$ is the pressure of ambient gas. For the adiabatic blastwave, $u$ is related to the shock velocity $u_{\mathrm{sh}}=$ $\frac{d R_{\text {sh }}}{d t}$ by the equation $u_{s h}=\frac{\gamma_{\mathrm{ad}}+1}{2} u$, where $\gamma_{\mathrm{ad}}$ is the ratio of the specific heats (adiabatic index). The energy of explosion $\mathcal{E}=\mathcal{E}_{\text {th }}+\frac{1}{2} M u^{2}$ consists of the internal energy $\mathcal{E}_{\text {th }}=\frac{4 \pi R_{\mathrm{sh}}^{3}}{3\left(\gamma_{\mathrm{ad}}-1\right)} P_{\text {in }}$ and the kinetic energy.
Now for a very strong shock where $P_{\text {in }}$ is negligible compared to $P$, Eq. (A2) can be presented as:

$$
\frac{\mathrm{d}(M u)^{2}}{\mathrm{~d} R_{\mathrm{sh}}}=\frac{12\left(\gamma_{\mathrm{ad}}-1\right)}{\left(\gamma_{\mathrm{ad}}+1\right) R_{\mathrm{sh}}}\left(\mathcal{E} M-\frac{1}{2}(M u)^{2}\right) .
$$

The solution of Eq. (A3) allows finding the shock velocity and the shock age as functions of the shock radius:

$$
\begin{aligned}
u_{\mathrm{sh}}\left(R_{\mathrm{sh}}\right) & =\frac{\gamma_{\mathrm{ad}}+1}{2}\left[\frac{2 w \mathcal{E}}{M^{2}\left(R_{\mathrm{sh}}\right) R_{\mathrm{sh}}^{w}} \int_{0}^{R_{\mathrm{sh}}} \mathrm{d} r r^{w-1} M(r)\right]^{1 / 2}, \\
t\left(R_{\mathrm{sh}}\right) & =\int_{0}^{R_{\mathrm{sh}}} \frac{\mathrm{d} r}{u_{\mathrm{sh}}(r)}
\end{aligned}
$$

where $w=\frac{6\left(\gamma_{\mathrm{ad}}-1\right)}{\gamma_{\mathrm{ad}}+1}$, which coincides with Eq. (17) in the main text.

\section{References}

Aharonian, F. A., Akhperjanian, A. G., Beilicke, M. et al. 2002, A\&A, 395,803

Axford, W. I. 1994, ApJS, 90, 937

Bell, A. R. 1978, MNRAS, 182, 147

Bell, A. R., \& Lucek, S. G. 2001, MNRAS, 321, 433

Berezhko, E. G., Ksenofontov, L. T., Ptuskin, V. S., Zirakashvili, V. N., \& Völk, H. J. 2003, A\&A 410, 189

Berezhko, E. G., \& Krymsky, G. F. 1988, Sov. Phys. Uspekhi, 31, 83

Berezhko, E. G., \& Völk, H. J. 2000, A\&A, 357, 283

Berezhko, E. G., \& Völk, H. J. 2003, Proc. 28th Intern. Cosmic Ray Conf., Tsukuba, 2433

Berezhko, E. G., Yelshin, V. K., \& Ksenofontov, L. T. 1996, JETP, 82, 1

Berezinskii, V. S., Bulanov, S. V., Ginzburg, V. L, Dogiel, V. A., \& Ptuskin, V. S. 1990, Astrophysics of Cosmic Rays, ed. V. L. Ginzburg, Amsterdam, North Holland

Berezinsky, V. S., Grigorieva, S. I.,\& Hnatyk, B. I. 2004, Astropart. Phys., 21, 617

Berezinsky, V. S., \& Ptuskin, V. S. 1989, A\&A 215, 399

Bisnovatyi-Kogan, G. S., \& Silich, S. A. 1995, Rev. Mod. Phys., 67, 661

Blandford, R. D., \& Ostriker, J. P. 1980, ApJ, 237, 793

Buckley, J. H., Akerlof, C. W., Carter-Lewis, D. A., et al. 1998, A\&A, 329,639

Bykov, A. M., \& Toptygin, I. N. 2001, Astron. Lett., 27, 625

Chevalier, R. A. 1982, ApJ, 259, 302

Chevalier, R. A., \& Liang, E. P. 1989, ApJ, 344, 332

Drury, L. O'C., Duffy, P., \& Kirk, J. G. 1996, ApJ, 309, 1002

Drury, L. O'C., Ellison, D. E., Aharonian, F. A., et al. 2001, Space Sci. Rev., 99, 329

Drury, L. O'C., van der Swaluw, E., \& Carroll, O. 2003, [arXiv: astro-ph/0309820]

Eichler, D. 1984, ApJ, 277, 429

Fedorenko, V. N. 1990, Preprint 1442, A. F. Ioffe Phys. Tech. Inst., Leningrad

Gaisser, T. K. et al. 1993, Phys. Rev. D47, 1919

Hörandel, J. R. 2003, Astroparticle Physics, 19, 193

Imshennik, V. S., Nadyozhin, D. K., \& Utrobin, V. P. 1981, Ap\&SS, 78, 105

Jones, F. C., Lukasiak, A., Ptuskin, V., \& Webber, W. 2001, ApJ, 547, 264 
Klepach, E. G., Ptuskin, V. S., \& Zirakashvili, V. N. 2000, Astropart. Phys., 13, 161

Krymsky, G. F. 1977, Soviet Physics-Doklady, 22, 327

Kulikov, G. V., \& Khristiansen, G. B. 1958, JETP 35, 441

Lozinskaya, T. A. 1992, Supernovae and Stellar Wind in the

Interstellar Medium, New York, American Institute of Physics

Malkov, M. A., \& Drury, L. O'C. 2001, Rep. Prog. Phys., 64, 429

Nadyozhin, D. K. 1981, Preprint ITEP, \#1

Nadyozhin, D. K. 1985, Ap\&SS, 112, 225

Ostriker, J. P., \& McKee, C. F. 1988, Rev. Mod. Phys., 60, 1

Parker, E. N. 1958, ApJ, 128, 664

Ptuskin, V. S. 2001, Space Sci. Rev., 99, 281

Ptuskin, V. S., Rogovaya, S. I., Zirakashvili, V. N., et al. 1993, A\&A, 268,726

Ptuskin, V. S., \& Zirakashvili, V. N. 2003, A\&A, 403, 1 (Paper I)

Roulet, E. 2004, Int. J. Mod. Phys. A, 19, 1133
Silberberg, R., Tsao, C. H., Shapiro, M. M., \& Biermann, P. L. 1991, in Cosmic Rays, Supernovae, Interstellar Medium, ed. M. M. Shapiro, R. Silberberg, \& J. P. Wefel (Kluwer Ac. Publ.), 97

Sveshnikova, L. G. 2003, A\&A, 409, 799

Truelove, J. K., \& McKee, C. F. 1999, ApJS, 120, 299

Ulrich, H., Antonia, T., Apel, W. D., et al. 2003, Nucl. Phys. B (Proc. Suppl.), 122, 218

Verma, M. K., Roberts, D. A., Goldstein, M. L., et al. 1996, JGR, 101, 21619

Vink, J. 2003 [arXiv: astro-ph/0311406]

Völk, H. J., \& Zirakashvili, V. N. 2004, A\&A, 417, 807

Völk, H. J., Zank, L. A., \& Zank, G. P. 1988, A\&A, 198, 274

Weaver, R., McCray, R., Castor, J., Shapiro, P., \& Moore, R. 1977, ApJ, 218, 377

Yoshida, T., \& Yanagita, S. 2001, Proc. 27th Intern. Cosmic Ray Conf., Hamburg, OG3.3.11 